Center for

Mathematical Economics

Working Papers

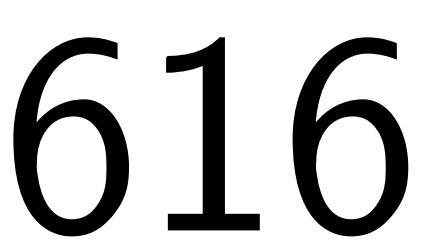

May 2019

\title{
Mortality Options: the Point of View of an Insurer
}

Maren Diane Schmeck and Hanspeter Schmidli

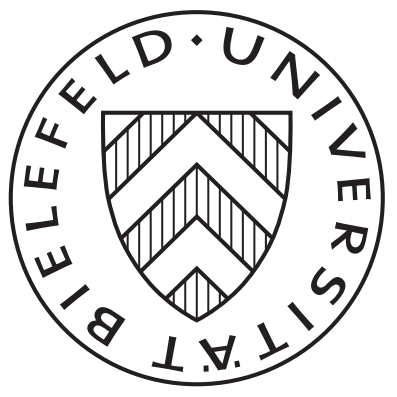

Center for Mathematical Economics (IMW) 


\title{
Mortality Options: the Point of View of an Insurer
}

\author{
Maren Diane Schmeck ${ }^{1}$ \\ Center for Mathematical Economics \\ Bielefeld University \\ PO Box 100131 \\ 33501 Bielefeld, Germany
}

\author{
HANSPETER SCHMidLI ${ }^{2}$ \\ Institute of Mathematics \\ University of Cologne \\ Weyertal 86-90 \\ 50931 Köln, Germany
}

May 24, 2019

\begin{abstract}
We consider the surplus process of a life insurer who is able to buy a securitisation product to hedge mortality in a discrete time framework. Two cohorts are considered: one underlying the securitisation product and one for the portfolio of the insurer. In our main result we show that there exists a unique strategy that maximises the expected utility of the insurer. Our findings are illustrated by a tractable model for mortality catastrophe risk.
\end{abstract}

Keywords: MORTALITY OPTION; OPTIMAL STRATEGY; MAXIMAL UTILITY; EXPONENTIAL UTILITY

Classification: Primary 91B30; secondary 93E20

\footnotetext{
${ }^{1}$ e-mail: maren.schmeck@uni-bielefeld.de

${ }^{2}$ e-mail: schmidli@math.uni-koeln.de
} 


\section{Introduction}

The basic idea behind insurance is to diversify and share risks. If a portfolio consists of many independent and identical risks, the law of large numbers implies that each customer will have to cover approximately the expected value of a single risk. The insured then pays a security loading together with the expected loss, the insurer pays the loss and takes the risk of extraordinary losses. In the average, the insurer will make a profit that plays the rôle of a compensation for overtaking the risk.

If a systematic risk is involved, the idea behind insurance does not work well any more. For example, severe hurricanes will apply to all the customers of a certain region. Since such a loss can be enormous, it may threaten the whole industry. In such a scenario the loss might be even too large for reinsurers. It was observed that financial markets easily could bare such an extreme risk. So the idea arose to transfer the insurance risk to the financial markets. Even though not perfectly matching the own portfolio, these securitisation products work similarly to a reinsurance contract. For an investor, the products yield a possibility to diversify an investment portfolio. Also insurers may act as an investor and in this way diversify their own risk to regions where they have not underwritten contracts and to hedge parts of their own portfolio via the securitisation product.

The literature on securitisation products either considers the point of view of an investor and/or a change to a risk neutral measure (e.g. [1, 6, 9, 12, 16, 17, 20, 22]), or the product is used to perform a Markovitz optimisation (e.g. [5, 7, 10, 15]). From the point of view of an insurer, this only partially answers the question how to choose a securitisation portfolio. The liability of an insurer is mainly determined by his own portfolio and therefore not completely matched by an index covering the losses of many companies. We will here use utility theory to determine the optimal portfolio, also taking consideration of the insurance risk. In order to simplify the presentation, we consider the case of a longevity securitisation product. Similar considerations would also apply for other securitisation products.

Let us start by a (non-comprehensive) overview on the literature on securitisation. One of the first products of this kind were the so called CAT Futures, see $[12,16]$. This was a future on an index built of catastrophic losses of certain insurance companies from catastrophes in a determined period reported to the insurer until a given deadline. Because of conceptual problems, they never became popular. The product was then replaced by PCS options. PCS, an agent working for the insurance industry, publishes regularly an index of the estimates of catastrophic losses. The indices are available for six different regions. Further, there are three national indices. The options are traded as spreads on the index. PCS options are treated in $[1,9,20,23]$.

Another possibility are securitisation bonds (SB). These are bonds where the interest and/or the principal are at risk. The reduction of the interest is triggered by well-defined events. Examples are the USAA hurricane bonds, the Winterthur Hail-Storm-Bonds, Swiss Re California Earthquake bonds, see [10, 11, 22]. 
The idea was also used for the securitisation of life insurance contracts. A first product were the Swiss Re mortality catastrophe bonds. The trigger for this bond was a weighted average of mortality rates over five countries. A second product was the EIB/BNP Paribas Longevity Bond. The trigger was a cohort of English and Welsh males. These products are described in [6].

As in the two examples mentioned above, mortality bonds are usually based on a mortality index. For example, J.P. Morgan developed their own mortality index called LifeMetrics. Later, many companies unified and founded the Life and Longevity Markets Association (LLMA) to share their knowledge and data in order to create a standardized foundation for the market. Among others AXA, Deutsche Bank, J.P. Morgan, Munich Re, Morgan Stanley, Swiss Re and UBS where members of the association. It aims at building a liquid trading market by developing a mortality index. For a specification of recently traded volume see e.g. the introduction of [18].

The financial engineering of these contracts is an involved issue, and can have non-trivial payoff structures. Here, we decided to assume a simple payoff structure being a one time payment at maturity $n$, which can be seen as building block for more complicated payoffs. We follow the same principle on the insurance side: we do not consider annuities, but insurance contracts with one time payoffs. Also the premium for the insurance is assumed to be a one time payment that is already included in the initial wealth. However, in a large portfolio the incoming premium is (almost) deterministic and in the case of a defined contribution pension plan, the liability at terminal wealth can be seen as the bookkeeping value of the future liabilities.

The SB can be issued by an insurer or reinsurer, as the Swiss Re mortality catastrophe bond. On the other hand, it can be issued by a financial market player as the EIB/BNP Paribas Longevity Bond. A further possibility is a Special Purpose Vehicle, often placed offshore by legal reasons, that issues the product for an insurer or reinsurer. In this paper, we will assume that the SB is issued by a financial institution and provides a hedge for the insurance industry. In this framework, we show that there exist a unique trading strategy maximizing the utility of terminal wealth. Then, we consider the case of an insurer, who faces liabilities from a pure endowment insurance, and who wants to hedge against catastrophic mortality risk.

In the literature, the modelling of mortality, as well as pricing and hedging of securitisation products gained a lot of attention, see e.g. $[2,4,5,13,14]$ and the references therein. In contrast, we decided here to follow a stochastic control approach. The insurer wants to hedge against systematic mortality risk, but by maximizing expected utility of the wealth at terminal time, that is at maturity of the insurance contracts. Related control problems in life insurance are concerned with the management of pension funds, which are essentially problems of asset allocation see e.g. $[3,7,15]$. Mostly, it is only allowed to trade into a risky asset and a riskless asset, but not into a securitisation product. Exceptions are [17] and [18]. 
We would like to mention another stream of literature, that factorizes insurance risk into risk factors as systematic and unsystematic risk, see [8, 19].

The rest of the paper is organised as follows: we will first introduce a simple model in discrete time for a securitisation index and losses for the insurance portfolio. We then show that a unique optimal strategy for the investment in a securitisation product exists. A very simple example illustrates the theoretical findings in the framework of pure endowment life insurance and catastrophic mortality risk.

\section{The model and the main result}

\subsection{Setting and problem formulation}

Let $\left\{I_{t}\right\}$ be an index modelling the portfolio of an insurer and $\left\{L_{t}\right\}$ be an external index underlying the securitisation product. In our context, the index describes the relative number of survivors in the reference portfolio. At time $n$, the insurer has the liability $f_{I}\left(I_{n}\right)$. One unit of the securitisation product will give a reward $L_{n}$. More specifically, $L_{t}$ models the number of survivors in the reference portfolio at time $t$. We have chosen this type of option to simplify the model. A reward $\mathbb{1}_{L_{n}>\kappa}$ for some $\kappa$ would work analogously but with less explicit expressions. At each time $0,1, \ldots, n-1$, the insurer can decide, how many units of the securitisation product he will have in his portfolio. The preferences of the insurer are determined by a utility function $u(w)$ that is strictly increasing and strictly concave. We assume that $\inf _{w} u^{\prime}(w)=\lim _{w \rightarrow \infty} u^{\prime}(w)=0$.

We now work on a probability space $(\Omega, \mathscr{F}, \mathbb{P})$. The information up to time $t$ is determined by a filtration $\left\{\mathscr{F}_{t}\right\}$. The processes $\left\{I_{k}\right\}$ and $\left\{L_{k}\right\}$ are adapted to this filtration. We assume that the reference portfolio consists of a homogeneous cohort of the same age, $x$ say, for example of females or males of age $x$ at time zero. The probability conditioned on $\mathscr{F}_{t}$ of an individual in the reference portfolio who is $y$ year old at time $t$ to survive for at least $m$ years is denoted by ${ }_{m} p_{y}(t)$. Because our estimates should be best estimates of the survival probabilities and therefore should not be biased, we must have $\mathbb{E}\left[{ }_{m} p_{y}(s) \mid \mathscr{F}_{t}\right]={ }_{m} p_{y}(t)$ for all $s \geq t$. Note that a mortality improvement like in a (non-stochastic) Lee-Carter model is already included in our basic mortality rates. The stochastic behaviour comes from future fluctuations of the mortality rates. We further assume that the interest rate $r$ is constant, that is the discounting factor for $m$ years is $(1+r)^{m}$. We assume that the price of a unit of the index is given by the conditional expectation of the discounted payoff. Conditional on $\mathscr{F}_{t}$, the number of survivors in the reference portfolio, $L_{n}$ is binomially distributed with parameters $L_{t}$ and ${ }_{n-t} p_{x+t}(t)$. Thus the price of a unit of the index at time $t \leq n$ is ${ }_{n-t} p_{x+t}(t)(1+r)^{-(n-t)} L_{t}$. In particular, this implies that ${ }_{m} p_{y}(t)$ is the survival probability under the pricing measure.

If $L_{0}=0$, then there will not be a possibility to invest in the mortality bond, 
and no decision has to be taken. We therefore assume that $L_{0} \neq 0$, and that $L_{1}$ is not deterministic.

The insurer starts with a wealth $W_{0}=w$. At time $k$, the insurer buys $\theta_{k}$ units of the mortality bond. Thus the wealth at time $k+1$ is then given by

$$
\begin{aligned}
& W_{k+1}=\left(W_{k}-\theta_{k} \frac{n-k}{p_{x+k}(k) L_{k}}(1+r)^{n-k}\right)(1+r)+\theta_{k} \frac{n-k-1}{p_{x+k+1}(k+1) L_{k+1}} \\
&=W_{k}(1+r)^{n-k-1} \\
&=\theta_{k} \frac{n-k-1}{p_{x+k+1}(k+1) L_{k+1}-{ }_{n-k} p_{x+k}(k) L_{k}} \\
&(1+r)^{n-k-1}
\end{aligned}
$$

Denote by

$$
V_{n}\left(w, x, I_{0}, L_{0}\right)=\sup _{\theta_{k}} \mathbb{E}\left[u\left(W_{n}-f_{I}\left(I_{n}\right)\right)\right]
$$

the maximal expected utility for a time horizon of length $n$. The variable $x$ denotes the underlying cohort. For the simplicity of the notation we let $V_{0}\left(w, x, I_{0}, L_{0}\right)=$ $u\left(w-f_{I}\left(I_{0}\right)\right)$. Then $V_{0}$ is a strictly increasing strictly concave function in the first argument.

Recursively, we get

$V_{n+1}\left(w, x, I_{0}, L_{0}\right)=\sup _{\theta} \mathbb{E}\left[V_{n}\left(w(1+r)+\theta \frac{{ }_{n} p_{x+1}(1) L_{1}-{ }_{n+1} p_{x}(0) L_{0}}{(1+r)^{n}}, x+1, I_{1}, L_{1}\right)\right]$.

This is the Bellman equation connected to our problem. For more literature on optimisation in insurance see for example [21] and the references therein.

\subsection{Existence and uniqueness of an optimal control}

In the following theorem we address existence and uniqueness of a solution to the just stated optimization problem. In particular, it turns out that we need to assume a condition of Inada type on the first derivative of $V_{n}$. In Proposition 1 we then show that in the above defined recursion, we stay in the class of functions that we consider. Thus, we show that there exists a unique trading strategy $\theta_{k}, k=1, \ldots, n$. Note that we have assumed that the condition on the first derivative holds for the utility function.

Theorem 1. Suppose that $V_{n}(w, x, I, L)$ is a strictly increasing and strictly concave function in $w$, and that $\lim _{w \rightarrow \infty} V_{n}^{\prime}(w, x, I, L)=0$. Then there is a unique $\theta_{0} \in \mathbb{R}$ such that

$$
V_{n+1}\left(w, x, I_{0}, L_{0}\right)=\mathbb{E}\left[V_{n}\left(w(1+r)+\theta_{0} \frac{{ }_{n} p_{x+1}(1) L_{1}-{ }_{n+1} p_{x}(0) L_{0}}{(1+r)^{n}}, x+1, I_{1}, L_{1}\right)\right] .
$$


Proof. We first show that the function

$$
\theta \mapsto \mathbb{E}\left[V_{n}\left(w(1+r)+\theta \frac{{ }_{n} p_{x+1}(1) L_{1}-{ }_{n+1} p_{x}(0) L_{0}}{(1+r)^{n}}, x+1, I_{1}, L_{1}\right)\right]
$$

is strictly concave in $\theta$. Let $\theta_{1}, \theta_{2} \in \mathbb{R}, \theta_{1} \neq \theta_{2}$, and $\alpha \in(0,1)$. Then for $\theta_{0}=$ $\alpha \theta_{1}+(1-\alpha) \theta_{2}$

$$
\begin{aligned}
\mathbb{E}\left[V_{n}\left(w(1+r)+\theta_{0} \frac{{ }_{n} p_{x+1}(1) L_{1}-{ }_{n+1} p_{x}(0) L_{0}}{(1+r)^{n}}, x+1, I_{1}, L_{1}\right)\right] \\
=\mathbb{E}\left[V _ { n } \left(\alpha\left\{w(1+r)+\theta_{1} \frac{{ }_{n} p_{x+1}(1) L_{1}-{ }_{n+1} p_{x}(0) L_{0}}{(1+r)^{n}}\right\}\right.\right. \\
\left.\left.+(1-\alpha)\left\{w(1+r)+\theta_{2} \frac{{ }_{n} p_{x+1}(1) L_{1}-{ }_{n+1} p_{x}(0) L_{0}}{(1+r)^{n}}\right\}, x+1, I_{1}, L_{1}\right)\right] \\
>\alpha \mathbb{E}\left[V_{n}\left(w(1+r)+\theta_{1} \frac{{ }_{n} p_{x+1}(1) L_{1}-{ }_{n+1} p_{x}(0) L_{0}}{(1+r)^{n}}, x+1, I_{1}, L_{1}\right)\right] \\
\quad+(1-\alpha) \mathbb{E}\left[V_{n}\left(w(1+r)+\theta_{2} \frac{{ }_{n} p_{x+1}(1) L_{1}-{ }_{n+1} p_{x}(0) L_{0}}{(1+r)^{n}}, x+1, I_{1}, L_{1}\right)\right] .
\end{aligned}
$$

Note that a concave function is differentiable almost everywhere. We thus need to find the zero of

$$
\mathbb{E}\left[\left\{{ }_{n} p_{x+1}(1) L_{1}-{ }_{n+1} p_{x}(0) L_{0}\right\} V_{n}^{\prime}\left(w(1+r)+\theta \frac{{ }_{n} p_{x+1}(1) L_{1}-{ }_{n+1} p_{x}(0) L_{0}}{(1+r)^{n}}, x+1, I_{1}, L_{1}\right)\right],
$$

where we interpret the derivative as one possible value between the derivative from the right and the derivative from the left at points where the function is not differentiable. In order to show that the optimal value is in the interior, we take the limits as $\theta \rightarrow \pm \infty$.

We write for $W_{1}=W_{1}(\theta)$

$$
\begin{aligned}
& \mathbb{E}\left[\left({ }_{n} p_{x+1}(1) L_{1}-{ }_{n+1} p_{x}(0) L_{0}\right) V_{n}^{\prime}\left(W_{1}, x+1, I_{1}, L_{1}\right)\right] \\
& =\mathbb{E}\left[\left({ }_{n} p_{x+1}(1) L_{1}-{ }_{n+1} p_{x}(0) L_{0}\right) V_{n}^{\prime}\left(W_{1}, x+1, I_{1}, L_{1}\right) \mathbb{I}_{n} p_{x+1}(1) L_{1}>_{n+1} p_{x}(0) L_{0}\right] \\
& \quad+\mathbb{E}\left[\left({ }_{n} p_{x+1}(1) L_{1}-{ }_{n+1} p_{x}(0) L_{0}\right) V_{n}^{\prime}\left(W_{1}, x+1, I_{1}, L_{1}\right) \mathbb{I}_{n} p_{x+1}(1) L_{1}<_{n+1} p_{x}(0) L_{0}\right]
\end{aligned}
$$

By monotone convergence, we can interchange limit and integration. Thus

$$
\begin{aligned}
\lim _{\theta \rightarrow \infty} \mathbb{E}[ & \left.\left({ }_{n} p_{x+1}(1) L_{1}-{ }_{n+1} p_{x}(0) L_{0}\right) V_{n}^{\prime}\left(W_{1}, x+1, I_{1}, L_{1}\right)\right] \\
= & \mathbb{E}\left[\left({ }_{n} p_{x+1}(1) L_{1}-{ }_{n+1} p_{x}(0) L_{0}\right) \inf _{w} V_{n}^{\prime}\left(w, x+1, I_{1}, L_{1}\right) \mathbb{I}_{n} p_{x+1}(1) L_{1}>_{n+1} p_{x}(0) L_{0}\right] \\
& \quad+\mathbb{E}\left[\left({ }_{n} p_{x+1}(1) L_{1}-{ }_{n+1} p_{x}(0) L_{0}\right) \sup _{w} V_{n}^{\prime}\left(w, x+1, I_{1}, L_{1}\right) \mathbb{I}_{n} p_{x+1}(1) L_{1}<_{n+1} p_{x}(0) L_{0}\right] \\
= & \mathbb{E}\left[\left({ }_{n} p_{x+1}(1) L_{1}-{ }_{n+1} p_{x}(0) L_{0}\right) \sup _{w} V_{n}^{\prime}\left(w, x+1, I_{1}, L_{1}\right) \mathbb{I}_{n} p_{x+1}(1) L_{1}<_{n+1} p_{x}(0) L_{0}\right] \\
< & 0 .
\end{aligned}
$$


It is no problem that the left hand side may become $-\infty$. In the same way,

$$
\begin{aligned}
\lim _{\theta \rightarrow-\infty} & \mathbb{E}\left[\left({ }_{n} p_{x+1}(1) L_{1}-{ }_{n+1} p_{x}(0) L_{0}\right) V_{n}^{\prime}\left(W_{1}, x+1, I_{1}, L_{1}\right)\right] \\
= & \mathbb{E}\left[\left({ }_{n} p_{x+1}(1) L_{1}-{ }_{n+1} p_{x}(0) L_{0}\right) \sup _{w} V_{n}^{\prime}\left(w, x+1, I_{1}, L_{1}\right) \mathbb{I}_{n} p_{x+1}(1) L_{1}>_{n+1} p_{x}(0) L_{0}\right] \\
& \quad+\mathbb{E}\left[\left({ }_{n} p_{x+1}(1) L_{1}-{ }_{n+1} p_{x}(0) L_{0}\right) \inf _{w} V_{n}^{\prime}\left(w, x+1, I_{1}, L_{1}\right) \mathbb{I}_{n} p_{x+1}(1) L_{1}<_{n+1} p_{x}(0) L_{0}\right] \\
= & \mathbb{E}\left[\left({ }_{n} p_{x+1}(1) L_{1}-{ }_{n+1} p_{x}(0) L_{0}\right) \sup _{w} V_{n}^{\prime}\left(w, x+1, I_{1}, L_{1}\right) \mathbb{I}_{n} p_{x+1}(1) L_{1}>_{n+1} p_{x}(0) L_{0}\right] \\
> & 0 .
\end{aligned}
$$

Since the derivative is monotone there must be a point where the derivative changes from positive to negative. At this point the maximum is attained.

In the following proposition we show that the recursively defined value functions stay in the class of strictly increasing and strictly concave functions with a first derivative tending to zero in the first argument. While the first two properties are more or less inherited, we have to circumvent some technical problems for the condition on the derivative.

Proposition 1. Suppose $V_{n}(w, x, I, L)$ is a strictly increasing and strictly concave function in $w$, and that $\lim _{w \rightarrow \infty} V_{n}^{\prime}(w, x, I, L)=0$. Then $V_{n+1}\left(w, x, I_{0}, L_{0}\right)$ is strictly increasing and strictly concave in $w$, and $\lim _{w \rightarrow \infty} V_{n}^{\prime}(w, x, I, L)=0$ for all $I$ and $L$.

Proof. That $V_{n+1}\left(w, x, I_{0}, L_{0}\right)$ is strictly increasing follows readily.

Let $w_{1}, w_{2} \in \mathbb{R}, w_{1} \neq w_{2}$. There exist $\theta_{1}, \theta_{2}$, such that

$$
V_{n+1}\left(w_{i}, x, I_{0}, L_{0}\right)=\mathbb{E}\left[V_{n}\left(w_{i}(1+r)+\theta_{i} \frac{{ }_{n} p_{x+1}(1) L_{1}-{ }_{n+1} p_{x}(0) L_{0}}{(1+r)^{n}}, x+1, I_{1}, L_{1}\right)\right] .
$$

Let $w_{0}=\alpha w_{1}+(1-\alpha) w_{2}$ for $\alpha \in(0,1)$, and $\theta_{0}=\alpha \theta_{1}+(1-\alpha) \theta_{2}$. We find

$$
\begin{aligned}
& V_{n+1}\left(w_{0}, x, I_{0}, L_{0}\right) \geq \mathbb{E}\left[V_{n}\left(w_{0}(1+r)+\theta_{0} \frac{{ }_{n} p_{x+1}(1) L_{1}-{ }_{n+1} p_{x}(0) L_{0}}{(1+r)^{n}}, x+1, I_{1}, L_{1}\right)\right] \\
&> \alpha \mathbb{E}\left[V_{n}\left(w_{1}(1+r)+\theta_{1} \frac{{ }_{n} p_{x+1}(1) L_{1}-{ }_{n+1} p_{x}(0) L_{0}}{(1+r)^{n}}, x+1, I_{1}, L_{1}\right)\right] \\
&+(1-\alpha) \mathbb{E}\left[V_{n}\left(w_{2}(1+r)+\theta_{2} \frac{{ }_{n} p_{x+1}(1) L_{1}-{ }_{n+1} p_{x}(0) L_{0}}{(1+r)^{n}}, x+1, I_{1}, L_{1}\right)\right] \\
&= \alpha V_{n+1}\left(w_{1}, x, I_{0}, L_{0}\right)+(1-\alpha) V_{n+1}\left(w_{2}, x, I_{0}, L_{0}\right) .
\end{aligned}
$$

This proves the concavity.

It remains to prove that the derivative tends to zero. Denote by $\theta(w)$ the argument for which the supremum for initial capital $w$ is attained. Let $w_{m}$ be a sequence 
tending to infinity and $\theta_{m}=\theta\left(w_{m}\right)$. Suppose there are infinitely many $\theta_{m} \geq 0$. By considering a subsequence, we can assume that $\theta_{m} \geq 0$ for all $m$. Choose $\kappa<0$, such that

$$
\mathbb{P}\left[{ }_{n} p_{x+1}(1) L_{1}-{ }_{n+1} p_{x}(0) L_{0}<\kappa\right]>0
$$

Let

$$
\gamma_{n}={ }_{n} p_{x+1}(1) L_{1}-{ }_{n+1} p_{x}(0) L_{0}
$$

We have

$$
\begin{aligned}
& 0= \mathbb{E}\left[\gamma_{n} V_{n}^{\prime}\left(w_{m}(1+r)+\theta_{m} \frac{\gamma_{n}}{(1+r)^{n}}, x+1, I_{1}, L_{1}\right)\right] \\
&=\mathbb{E}\left[\mathbb{I}_{\gamma_{n} \geq 0} \gamma_{n} V_{n}^{\prime}\left(w_{m}(1+r)+\theta_{m} \frac{\gamma_{n}}{(1+r)^{n}}, x+1, I_{1}, L_{1}\right)\right] \\
& \quad+\mathbb{E}\left[\mathbb{I}_{\kappa<\gamma_{n}<0} \gamma_{n} V_{n}^{\prime}\left(w_{m}(1+r)+\theta_{m} \frac{\gamma_{n}}{(1+r)^{n}}, x+1, I_{1}, L_{1}\right)\right] \\
&+\mathbb{E}\left[\mathbb{I}_{\gamma_{n} \leq \kappa} \gamma_{n} V_{n}^{\prime}\left(w_{m}(1+r)+\theta_{m} \frac{\gamma_{n}}{(1+r)^{n}}, x+1, I_{1}, L_{1}\right)\right] .
\end{aligned}
$$

By monotone convergence we find

$$
\begin{aligned}
0 & \leq \lim _{m \rightarrow \infty} \mathbb{E}\left[\mathbb{I}_{\gamma_{n} \geq 0} \gamma_{n} V_{n}^{\prime}\left(w_{m}(1+r)+\theta_{m} \frac{\gamma_{n}}{(1+r)^{n}}, x+1, I_{1}, L_{1}\right)\right] \\
& \leq \lim _{m \rightarrow \infty} \mathbb{E}\left[\mathbb{I}_{\gamma_{n} \geq 0} \gamma_{n} V_{n}^{\prime}\left(w_{m}(1+r), x+1, I_{1}, L_{1}\right)\right]=0 .
\end{aligned}
$$

Thus the other two terms also have to converge to zero. Clearly,

$$
\lim _{m \rightarrow \infty} \mathbb{E}\left[\mathbb{I}_{\gamma_{n} \geq 0} V_{n}^{\prime}\left(w_{m}(1+r)+\theta_{m} \frac{\gamma_{n}}{(1+r)^{n}}, x+1, I_{1}, L_{1}\right)\right]=0
$$

Further,

$$
\begin{aligned}
0 & \leq \lim _{m \rightarrow \infty} \mathbb{E}\left[\mathbb{I}_{\gamma_{n} \leq \kappa} V_{n}^{\prime}\left(w_{m}(1+r)+\theta_{m} \frac{\gamma_{n}}{(1+r)^{n}}, x+1, I_{1}, L_{1}\right)\right] \\
& \leq \kappa^{-1} \lim _{m \rightarrow \infty} \mathbb{E}\left[\mathbb{I}_{\gamma_{n} \leq \kappa} \gamma_{n} V_{n}^{\prime}\left(w_{m}(1+r)+\theta_{m} \frac{\gamma_{n}}{(1+r)^{n}}, x+1, I_{1}, L_{1}\right)\right]=0 .
\end{aligned}
$$

This implies also

$$
\begin{aligned}
0 & \leq \lim _{m \rightarrow \infty} \mathbb{E}\left[\mathbb{I}_{\gamma_{n} \leq \kappa} V_{n}^{\prime}\left(w_{m}(1+r)+\theta_{m} \frac{\kappa}{(1+r)^{n}}, x+1, I_{1}, L_{1}\right)\right] \\
& \leq \lim _{m \rightarrow \infty} \mathbb{E}\left[\mathbb{I}_{\gamma_{n} \leq \kappa} V_{n}^{\prime}\left(w_{m}(1+r)+\theta_{m} \frac{\gamma_{n}}{(1+r)^{n}}, x+1, I_{1}, L_{1}\right)\right]=0
\end{aligned}
$$

and therefore $\lim \inf w_{m}(1+r)+\theta_{m} \frac{\gamma_{n}}{(1+r)^{n}}>-\infty$. This implies, that also

$$
\begin{aligned}
0 & \leq \lim _{m \rightarrow \infty} \mathbb{E}\left[\mathbb{I}_{\kappa<\gamma_{n}<0} V_{n}^{\prime}\left(w_{m}(1+r)+\theta_{m} \frac{\gamma_{n}}{(1+r)^{n}}, x+1, I_{1}, L_{1}\right)\right] \\
& \leq \lim _{m \rightarrow \infty} \mathbb{E}\left[\mathbb{I}_{\kappa<\gamma_{n}<0} V_{n}^{\prime}\left(w_{m}(1+r)+\theta_{m} \frac{\kappa}{(1+r)^{n}}, x+1, I_{1}, L_{1}\right)\right]=0 .
\end{aligned}
$$

Putting the limits together, shows that $\lim _{w \rightarrow \infty} V_{n+1}^{\prime}(w)=0$. An analogous proof applies if there are infinitely many $\theta_{m}<0$. 


\section{An example: catastrophe mortality risk}

In order to illustrate our theory, we consider a very simple model for future mortality. Our goal is not to have a realistic behaviour, but to be able to calculate the strategy easily. We will also propose a linear approximation. The simple model allows us easily to compare approximations to the complete model. For more realistic mortality models, the approach would be the same, but the numerical calculations will be more elaborate.

In this example, we consider a pure endowment life insurance. That is, each individual receives a payout of 1 if it survives until final time $n$. The liability of the insurer is then $f_{I}(I)=I$, as $I_{n}$ denotes the number of survivors in the portfolio of the insurer. Furthermore, we have to model the development of the indices. In order to keep the model simple, we do not consider longevity risk, but aim at modelling some sort of 'catastrophic' mortality events. One could think for example of an epidemic (influenza, cholera, ...) or on extreme weather conditions: during a very hot summer or a very cold winter, more people die. On the other hand, also an improvement of the mortality is possible for one year. We will assume that these events happen independently from each other: e.g., a hot summer last year does not influence the weather conditions of this year. To obtain a simple model, the events do not influence future mortality, so that our initial assumptions are not updated. The shock in period $n$, thus, influences the number of survivors in period $n$, only, and not the future mortality rates. For example, our mortalities may take the possibility of an influenza pandemic into account. But before period $n$ we do not know whether the pandemic took place or not.

At time $s<t$ the best prior estimate of the probability of death for a member of the cohort in the period $(t-1, t]$ is ${ }_{1} q_{x+t-1}(0)$. At time $t$, the realised death probability is ${ }_{1} q_{x+t-1}(t)={ }_{1} q_{x+t-1}(0) Z_{t}$, where $\left\{Z_{t}\right\}$ are iid positive variables with expected value 1 . We assume in addition that ${ }_{1} q_{x+t-1}(t) \leq 1$. That means that the variables $Z_{t}$ are bounded and that the mortalities ${ }_{1} q_{x+t-1}(0)$ are bounded away from 1. The filtration is the natural filtration of the variables $\left\{Z_{t}\right\}, \mathscr{F}_{t}=\sigma\left(Z_{k}: 1 \leq\right.$ $k \leq t)$. We have $\mathbb{E}\left[{ }_{1} q_{x+t-1}(t) \mid \mathscr{F}_{t-1}\right]={ }_{1} q_{x+t-1}(0)$, and therefore also $\mathbb{E}\left[{ }_{1} p_{x+t-1}(t) \mid\right.$ $\left.\mathscr{F}_{t-1}\right]={ }_{1} p_{x+t-1}(0)$ is the best estimate for the survival probability at time $t-1$. Looking at the period $(0, n]$, the expected value for survival for $n$ years is ${ }_{n} p_{x}(0)$, the best estimate at time 0 . The liability of the insurer is $f_{I}(I)=I$. That is, $I_{n}$ denotes the number of survivors in the portfolio of the insurer. The calculations below could be simplified if we had chosen the model ${ }_{1} p_{x+t-1}(t)={ }_{1} p_{x+t-1}(0) Z_{t}$, but then in our setup we had to choose ${ }_{1} p_{x+t-1}(0) \leq \frac{2}{3}$ (see below). This would lead to a very high mortality rate. Since pure endowment life insurance is more appropriate for not very old individuals, the assumption ${ }_{1} q_{x+t-1}(0) \leq \frac{2}{3}$ seems more reasonable.

Consider the probability ${ }_{n-s+1} p_{x+s}($.$) that an individual survives the time interval$ $[s, n+1]$. Before the period starts, for $t \leq s$, we assume that the best estimates are ${ }_{n-s+1} p_{x+s}(t)={ }_{n-s+1} p_{x+s}(0)$. After the start of the interval, for $t>s$, we adjust the 


\begin{tabular}{|l||c|c|c|}
\hline Period & 0 & 1 & 2 \\
\hline Age & $x$ & $x+1$ & $x+2$ \\
\hline${ }_{n+1} p_{x}(\cdot)$ & ${ }_{n+1} p_{x}(0)$ & $1-\left(1-{ }_{n+1} p_{x}(0)\right) Z_{1}$ & $1-\left(\left(1-{ }_{n+1} p_{x}(0)\right) Z_{1}\right) Z_{2}$ \\
${ }_{n} p_{x+1}(\cdot)$ & ${ }_{n} p_{x+1}(0)$ & ${ }_{n} p_{x+1}(0)$ & $1-\left(1-{ }_{n} p_{x+1}(0)\right) Z_{2}$ \\
${ }_{n-1} p_{x+2}(\cdot)$ & ${ }_{n-1} p_{x+2}(0)$ & ${ }_{n-1} p_{x+2}(0)$ & ${ }_{n-1} p_{x+2}(0)$ \\
\hline
\end{tabular}

Table 1: Best estimates for the mortalities

estimates for the realized number of dead individuals through the iid shocks $Z_{i}$. In Table 1 we have written down the corresponding expressions for $s=0,1,2$.

Suppose the value function $V_{n}(w, x, I, L)$ is known. We consider now a securitisation product with payoff in $n+1$. The value of the wealth process at time 1 reads

$$
W_{1}=W_{0}(1+r)+\theta_{0} \frac{{ }_{n} p_{x+1}(1) L_{1}-{ }_{n+1} p_{x}(0) L_{0}}{(1+r)^{n}} .
$$

Consider the index $L$. Because we assume a large portfolio, we can model the index as $L_{n+1}=L_{n 1} p_{x+n}(n+1)=L_{n}\left(1-\left(1-{ }_{1} p_{x+n}(0)\right) Z_{n+1}\right)$. This yields in particular

$$
L_{1}={ }_{1} p_{x}(1) L_{0}=\left(1-\left(1-{ }_{1} p_{x}(0)\right) Z_{1}\right) L_{0} \text {. }
$$

Thus

$$
\begin{aligned}
{ }_{n} p_{x+1}(1) L_{1}-{ }_{n+1} p_{x}(0) L_{0} & ={ }_{n} p_{x+1}(0)\left(1-\left(1-{ }_{1} p_{x}(0)\right) Z_{1}\right) L_{0}-{ }_{n+1} p_{x}(0) L_{0} \\
& =\left(1-Z_{1}\right)\left({ }_{n} p_{x+1}(0)-{ }_{n+1} p_{x}(0)\right) L_{0}
\end{aligned}
$$

where we used that ${ }_{n} p_{x+1}(1)={ }_{n} p_{x+1}(0)$ since the left hand side is the best estimate for the future. Because ${ }_{n} p_{x+1}$ here models a survival probability when the cohort is $x+1$ years old (that is from time 1 on), we get the first information on the realised mortality in period 2 , see also Table 1 . Note that ${ }_{n} p_{x+1}(0)>_{n+1} p_{x}(0)$. We assume that $I_{n+1}=I_{n}\left(1-\left(1-{ }_{1} p_{x+n}(0)\right) \tilde{Z}_{n+1}\right)$, also assuming a large portfolio. Then the process $I$ behaves in the same way as $L$ but with different random variables.

Consider the exponential utility function $u(x)=-\mathrm{e}^{-\alpha x}$ for some $\alpha>0$. We claim that $V_{n}(w, x, I, L)=-\exp \left\{-\alpha_{n} w+f_{n}(x, I, L)\right\}$ for some function $f_{n}$ and $\alpha_{n}=\alpha(1+r)^{n}$. We find by induction

$$
\begin{aligned}
& V_{n+1}(w, x, I, L) \mathrm{e}^{\alpha_{n} w(1+r)} \\
& \quad=\sup _{\theta}-\mathbb{E}\left[\exp \left\{-\alpha_{n} \theta \frac{\left(1-Z_{1}\right)\left({ }_{n} p_{x+1}(0)-{ }_{n+1} p_{x}(0)\right) L}{(1+r)^{n}}+f_{n}\left(x+1, I_{1}, L_{1}\right)\right\}\right] \\
& =-\inf _{\theta} \mathbb{E}\left[\exp \left\{-\alpha_{n} \theta \frac{\left(1-Z_{1}\right)\left({ }_{n} p_{x+1}(0)-{ }_{n+1} p_{x}(0)\right) L}{(1+r)^{n}}+f_{n}\left(x+1, I_{1}, L_{1}\right)\right\}\right] .
\end{aligned}
$$

We have $\alpha_{n+1}=\alpha_{n}(1+r)=\alpha(1+r)^{n+1}$ and, with $I_{1}=\left(1-\left(1-{ }_{1} p_{x}(0)\right) \widetilde{Z}_{1}\right) I$ and 


$$
\begin{aligned}
& L_{1}=\left(1-\left(1-{ }_{1} p_{x}(0)\right) Z_{1}\right) L, \\
& f_{n+1}(x, I, L) \\
& \quad=\inf _{\theta} \log \mathbb{E}\left[\exp \left\{-\alpha_{n} \theta \frac{\left(1-Z_{1}\right)\left({ }_{n} p_{x+1}(0)-{ }_{n+1} p_{x}(0)\right) L}{(1+r)^{n}}+f_{n}\left(x+1, I_{1}, L_{1}\right)\right\}\right] \\
& \quad=\inf _{\theta} \log \mathbb{E}\left[\exp \left\{-\alpha \theta\left(1-Z_{1}\right)\left({ }_{n} p_{x+1}(0)-{ }_{n+1} p_{x}(0)\right) L+f_{n}\left(x+1, I_{1}, L_{1}\right)\right\}\right] .
\end{aligned}
$$

Since $V_{0}(x)=-\mathrm{e}^{-\alpha\left(w-I_{0}\right)}$, the form is proven. Thus, the initial wealth is not relevant for the optimal strategy in this modelling framework, which is to be expected for the exponential utility function.

As a specific model, assume that

$$
\widetilde{Z}_{1}=\gamma U_{1}+(1-\gamma) Z_{1}
$$

where $Z_{1}, U_{1} \sim \mathcal{U}\left[\frac{1}{2}, \frac{3}{2}\right]$ are independent and $\gamma \in(0,1)$. As we assume a linear dependence between $\widetilde{Z}$ and $Z$, its dependence structure is determined by its correlation coefficient being equal to $1-\gamma$. Then also the dependence structure between $I_{1}$ and $L_{1}$ is described by its correlation, being $1-\gamma$ as well. Note that we allow for positive dependence only.

\subsection{One time step before maturity of the contracts}

After stating the relevant modelling assumptions, we use the relation (1) to calculate the optimal strategy and optimal function $f_{1}(x, I, L)$. That is, the insurer is one time step before maturity of the insurance contract. We get $\alpha_{0}=\alpha$ and $f_{0}(I)=\alpha I$. Noting ${ }_{0} p_{x+1}(0)=1$, we obtain

$$
\begin{aligned}
& f_{1}(x, I, L) \\
& \left.=\inf _{\theta} \log \mathbb{E}\left[\exp \left\{-\alpha \theta(1-Z)\left({ }_{0} p_{x+1}(0)-{ }_{1} p_{x}(0)\right) L+f_{0}\left(1-\left(1-{ }_{1} p_{x}(0)\right) \widetilde{Z}\right) I\right)\right\}\right] \\
& =\inf _{\theta} \log \mathbb{E}\left[\exp \left\{-\alpha \theta(1-Z)\left(1-{ }_{1} p_{x}(0)\right) L+\alpha\left(1-\left(1-{ }_{1} p_{x}(0)\right) \widetilde{Z}\right) I\right\}\right] \\
& \left.=\alpha_{1} p_{x}(0) I+\inf _{\theta} \log \mathbb{E}\left[\exp \left\{\alpha\left(1-{ }_{1} p_{x}(0)\right)[\theta L-(1-\gamma) I](Z-1)\right\}\right]\right\} \\
& \quad+\log \mathbb{E}\left[\exp \left\{\alpha\left(1-{ }_{1} p_{x}(0)\right) \gamma I(1-U)\right\}\right] \\
& =\alpha_{1} p_{x}(0) I+\log \frac{\sinh \left(\frac{1}{2} \alpha\left(1-{ }_{1} p_{x}(0)\right) \gamma I\right)}{\frac{1}{2} \alpha\left(1-{ }_{1} p_{x}(0)\right) \gamma I} \\
& \quad+\inf _{\theta} \log \frac{\sinh \left(\frac{1}{2} \alpha\left(1-{ }_{1} p_{x}(0)\right)[\theta L-(1-\gamma) I]\right)}{\frac{1}{2} \alpha\left(1-{ }_{1} p_{x}(0)\right)[\theta L-(1-\gamma) I]} .
\end{aligned}
$$

Now, $g(x)=\frac{1}{x} \sinh (x)$ takes its unique minimum in $x=0$ with $g(0)=1$. Thus,

$$
\theta^{*}:=(1-\gamma) \frac{I}{L}
$$


and

$$
f_{1}(x, I, L)=\alpha_{1} p_{x}(0) I+\log \frac{\sinh \left(\frac{1}{2} \alpha\left(1-{ }_{1} p_{x}(0)\right) \gamma I\right)}{\frac{1}{2} \alpha\left(1-{ }_{1} p_{x}(0)\right) \gamma I} .
$$

Note that $f_{1}$ does not depend on $L$. Because part of the mortality in the reference portfolio changes as the mortality in the own portfolio, the strategy is to hedge this dependent part of possible survivors with the securitisation product. Therefore, the form of our optimal portfolio is due to the simple model we use. Note that, if we replace $L$ by $\zeta L$, we can get the same value by choosing $\theta / \zeta$. Therefore, a $\theta$ proportional to $1 / L$ is to be expected.

\subsection{Two time steps before maturity of the contracts}

Taking into account that $f_{1}$ does not depend on $L$ we have that

$$
\begin{aligned}
f_{2}(x, I, L)= & \inf _{\theta} \log \mathbb{E}\left[\exp \left\{-\alpha \theta(1-Z)\left({ }_{1} p_{x+1}(0)-{ }_{2} p_{x}(0)\right) L+f_{1}\left(x+1, I_{1}, 0\right)\right\}\right] \\
= & \inf _{\theta} \log \mathbb{E}\left[\operatorname { e x p } \left\{-\alpha \theta(1-Z)\left({ }_{1} p_{x+1}(0)-{ }_{2} p_{x}(0)\right) L\right.\right. \\
& \left.\left.\quad+\alpha{ }_{1} p_{x+1}(0) I_{1}\right\} \frac{\sinh \left(\frac{1}{2} \alpha\left(1-{ }_{1} p_{x+1}(0)\right) \gamma I_{1}\right)}{\frac{1}{2} \alpha\left(1-{ }_{1} p_{x+1}(0)\right) \gamma I_{1}}\right] .
\end{aligned}
$$

where we have used the relation $(1)$ and $I_{1}=\left(1-\left(1-{ }_{1} p_{x}(0)\right) \widetilde{Z}\right) I$, where again $\widetilde{Z}=(1-\gamma) Z+\gamma U$ and $Z, U \sim \mathcal{U}\left[\frac{1}{2}, \frac{3}{2}\right]$. Note that the expectation on the right hand side does not have an explicit analytical representation. Nevertheless, we would like to illustrate its behaviour numerically.

\subsubsection{Numerical evaluation of the optimal position in the securitisation product two time steps before maturity of the insurance contract}

In the above expectation, we would have to evaluate numerically the expectation over three random variables, so a threefold integral. To simplify the numerical evaluation, we note first that

$$
\begin{aligned}
f_{2}(x, I, L)=\inf _{\theta} \log \mathbb{E}[ & \exp \left\{-\alpha \theta(1-Z)\left({ }_{1} p_{x+1}(0)-{ }_{2} p_{x}(0)\right) L\right. \\
& \left.\left.+\alpha\left[{ }_{1} p_{x+1}(0)+\left(1-{ }_{1} p_{x+1}(0)\right) \gamma(1-\widetilde{U})\right] I_{1}\right\}\right],
\end{aligned}
$$

for a random variable $\widetilde{U} \sim \mathcal{U}\left[\frac{1}{2}, \frac{3}{2}\right]$ being independent of $Z$ and $U$. Note that we can write

$$
I_{1}=\left[{ }_{1} p_{x}(0)+(1-\gamma)\left(1-{ }_{1} p_{x}(0)\right)(1-Z)+\gamma\left(1-{ }_{1} p_{x}(0)\right)(1-U)\right] I .
$$


Then

$$
\begin{aligned}
f_{2}(x, I, L)= & \inf _{\theta} \log \mathbb{E}\left[\operatorname { e x p } \left\{-\alpha \theta\left({ }_{1} p_{x+1}(0)-{ }_{2} p_{x}(0)\right)(1-Z) L\right.\right. \\
& +\alpha(1-\gamma)\left(1-{ }_{1} p_{x}(0)\right)\left[{ }_{1} p_{x+1}(0)+\left(1-{ }_{1} p_{x+1}(0)\right) \gamma(1-\widetilde{U})\right](1-Z) I \\
& +\alpha \gamma\left(1-{ }_{1} p_{x}(0)\right)\left[{ }_{1} p_{x+1}(0)+\left(1-{ }_{1} p_{x+1}(0)\right) \gamma(1-\widetilde{U})\right](1-U) I \\
& \left.\left.+\alpha{ }_{1} p_{x}(0)\left[{ }_{1} p_{x+1}(0)+\left(1-{ }_{1} p_{x+1}(0)\right) \gamma(1-\widetilde{U})\right] I\right\}\right] .
\end{aligned}
$$

Evaluating the expectation of the independent random variables $1-Z$ and $1-U$ results in

$$
f_{2}(x, I, L)=\alpha_{1} p_{x}(0){ }_{1} p_{x+1}(0) I+\inf _{\theta} f_{2}^{\theta}(x, I, L)
$$

where

$$
\begin{aligned}
f_{2}^{\theta}(x, I, L)=\log \mathbb{E}[ & \frac{\sinh \left(\frac{1}{2} r_{Z}(\theta, I, L, 1-\widetilde{U})\right)}{\frac{1}{2} r_{Z}(\theta, I, L, 1-\widetilde{U})} \frac{\sinh \left(\frac{1}{2} r_{U}(I, 1-\widetilde{U})\right)}{\frac{1}{2} r_{U}(I, 1-\widetilde{U})} \\
& \left.\times \exp \left\{\alpha_{1} p_{x}(0)\left(1-{ }_{1} p_{x+1}(0)\right) \gamma(1-\widetilde{U}) I\right\}\right]
\end{aligned}
$$

and

$$
\begin{aligned}
r_{Z}(\theta, I, L, 1-\widetilde{U})= & \alpha(1-\gamma)\left(1-{ }_{1} p_{x}(0)\right)\left[{ }_{1} p_{x+1}(0)+\left(1-{ }_{1} p_{x+1}(0)\right) \gamma(1-\widetilde{U})\right] I \\
& -\alpha \theta\left({ }_{1} p_{x+1}-{ }_{2} p_{x}(0)\right) L \\
r_{U}(I, 1-\widetilde{U})= & \alpha \gamma\left(1-{ }_{1} p_{x}(0)\right)\left[{ }_{1} p_{x+1}(0)+\left(1-{ }_{1} p_{x+1}(0)\right) \gamma(1-\widetilde{U})\right] I .
\end{aligned}
$$

Here we have to evaluate only the expectation of the random variable $1-\widetilde{U}$.

This is illustrated in Figures 1 and 2, where we determine $f_{2}$ and $\theta^{*}$ numerically for the parameters $\gamma=0.5,{ }_{1} p_{x}(0)=0.99,{ }_{1} p_{x+1}(0)=0.95,{ }_{2} p_{x}(0)=$ ${ }_{1} p_{x}(0){ }_{1} p_{x+1}(0)=0.9405$ and $\alpha=0.5$. The four panels of Figure 1 show the function $\theta \mapsto f_{2}^{\theta}(I, L)$ for $L=8, \ldots, 11$. In each panel, we plot one curve for $I=1, \ldots, 11$. It illustrates the location of the $\theta^{*}$ minimizing $f_{2}^{\theta}(I, L)$, being increasing if $I$ increases.

The first panel in Figure 2 shows the resulting optimal function $f_{2}(I, L)$. As in the first time step, it is independent of $L$. For comparison we have added $f_{1}(I, L)$ (dashed line). Note that we have added the subscripts to the $\theta$ if we want to emphasize that $\theta_{2}$ is a position to hold if two time steps are left, and $\theta_{1}$ is the position to hold if only one time step is left. The last panel shows $\theta_{2}^{*}$ as a function of $I$, note the rather linear shape. Again for comparison, we have added the optimal strategy $\theta_{1}^{*}$ (dashed line) if only one unit of time is left before the insurance product is paid out. The lines seem to be identical.

The optimal strategy seems to be still quite linear in $I$, but one can show that it is not. In the next section, we approximate the expression in the expectation of (4), such that the minimum can be found analytically and such that the approximated optimal strategy is indeed linear in $I$. 

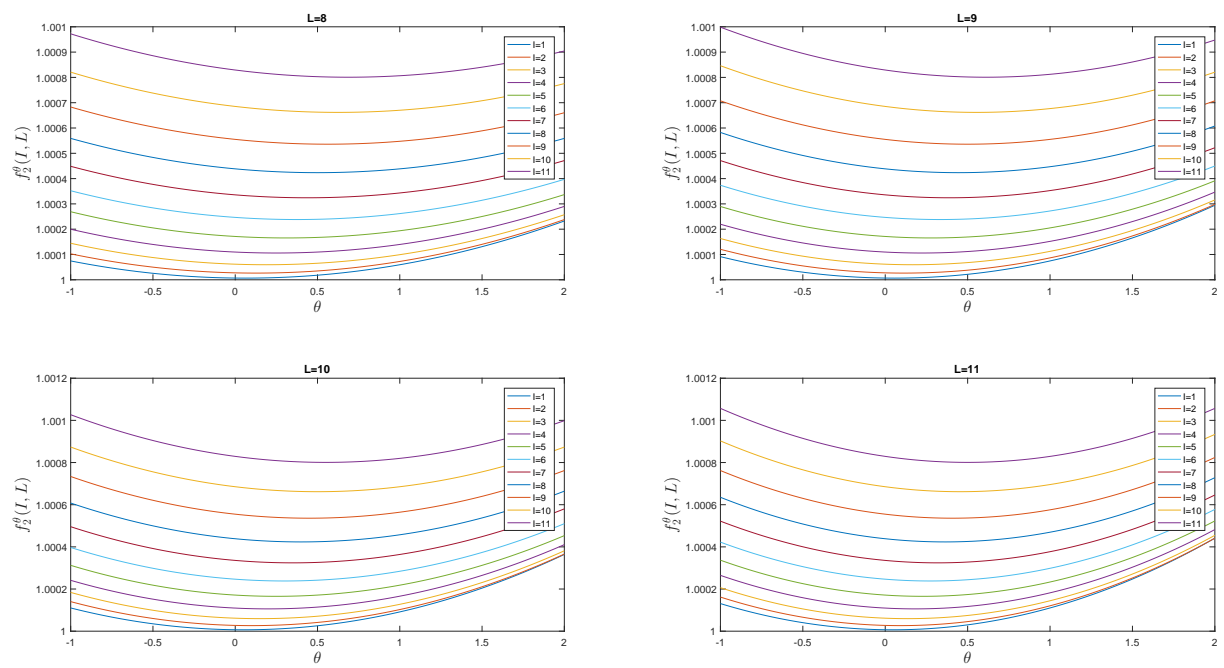

Figure 1: The function $f_{2}^{\theta}(I, L)$ which has to be minimized over $\theta$ for $L=8, \ldots, 11$ and $I=1, \ldots, 11$.
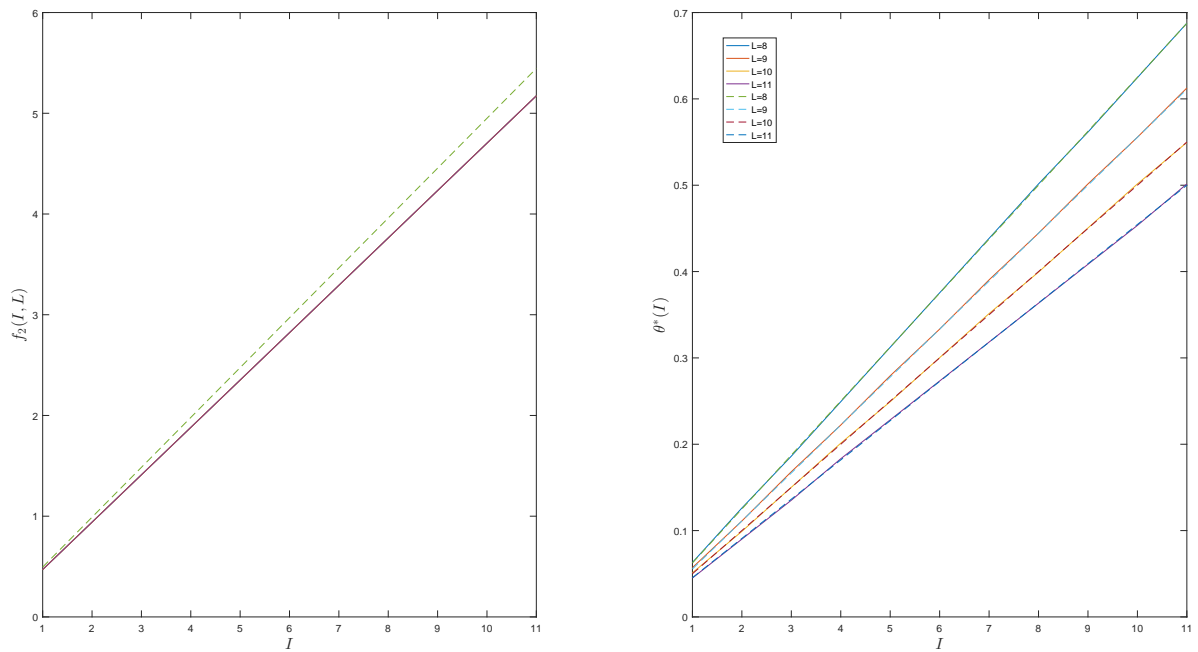

Figure 2: Left: The optimal functions $f_{2}(I, L)$ (solid line) and $f_{1}(I, L)$ (dashed). Right: The optimal strategies $\theta_{2}^{*}$ (solid line) and $\theta_{1}^{*}$ (dashed) if there are two and one time step left before maturity of the products. 


\subsubsection{Approximation of the optimal strategy two time steps before ma- turity}

Consider

$$
f_{2}(x, I, L)=\alpha_{1} p_{x}(0){ }_{1} p_{x+1}(0) I+\inf _{\theta} f_{2}^{\theta}(x, I, L)
$$

as defined in (4). Then

$$
f_{2}^{\theta}(x, I, L)=\log \mathbb{E}[g(\theta, \widetilde{U})]
$$

where

$$
\begin{aligned}
g(\theta, u)=\frac{\sinh \left(\frac{1}{2} r_{Z}(\theta, I, L, 1-u)\right)}{\frac{1}{2} r_{Z}(\theta, I, L, 1-u)} \frac{\sinh \left(\frac{1}{2} r_{U}(I, 1-u)\right)}{\frac{1}{2} r_{U}(I, 1-u)} \\
\quad \times \exp \left\{\alpha \gamma_{1} p_{x+1}(0)\left(1-{ }_{1} p_{x}(0)\right)(1-u) I\right\} .
\end{aligned}
$$

The random variable $\widetilde{U}$ is uniformly distributed with $\mathbb{E}[\widetilde{U}]=1$. Thus, motivated by Figure 2, linearising $g$ by a first order Taylor expansion around $u=1$ yields

$$
g(\theta, u) \approx g(\theta, 1)+g_{u}(\theta, 1)(u-1) .
$$

Then

$$
f_{2}^{\theta}(x, I, L) \approx g(\theta, 1)+g_{u}(\theta, 1) \mathbb{E}[\widetilde{U}-1]=g(\theta, 1) .
$$

Now, $g(\theta, 1)$ attains the minimum in $r_{Z}(\theta, I, L, 0)=0$, that is

$$
\theta_{\text {appr }}^{*}=(1-\gamma) \frac{{ }_{1} p_{x+1}(0)\left(1-{ }_{1} p_{x}(0)\right)}{{ }_{1} p_{x+1}(0)-{ }_{2} p_{x}(0)} \frac{I}{L}
$$

As ${ }_{1} p_{x+1}(0){ }_{1} p_{x}(0)={ }_{2} p_{x}(0)$ we have that ${ }_{1} p_{x+1}(0)\left(1-{ }_{1} p_{x}(0)\right)={ }_{1} p_{x+1}(0)-{ }_{2} p_{x}(0)$ and thus

$$
\theta_{\text {appr }}^{*}=(1-\gamma) \frac{I}{L}
$$

being equal to (2). In Figure 3 the numerical optimal strategy $\theta_{2}^{*}$ is shown, as well the approximated strategy $\theta_{a p p r}^{*}$ above (circles). The approximation seems to be amazingly exact.

\section{Conclusion}

If systematic risk is involved, the classical principle of insurance does not work any more. Nevertheless, it is possible to transfer parts of this systematic insurance risk to the financial market using so-called securitisation products. In this paper, we take the view of a life insurance company, that can hedge its claims by trading 


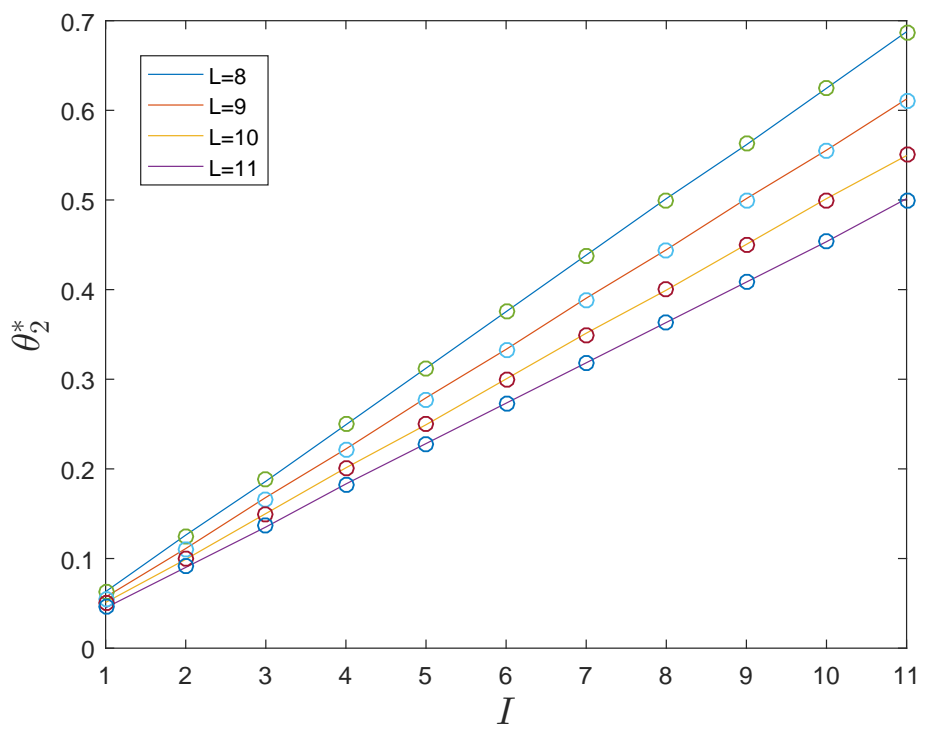

Figure 3: The numerically determined optimal strategy $\theta_{2}^{*}$ (straight line) and its approximation $\theta_{\text {appr }}^{*}$ being linear in $I$ (circles).

in discrete time into a longevity securitisation product. The goal is to maximize the expected utility of terminal wealth. Using the Bellman principle, we show in a rather general framework that a unique optimal strategy exists and that it is unique. It turns out that we have to make some assumptions on the value function, which we show are fulfilled in each step of our defined recursion. In an toy example we illustrate how to calculate the optimal strategy recursively for a short term horizon. In the same way, realistic models for the stochastic behaviour of mortality can be treated. The method would also work for other lines of business and the corresponding securitisation products.

\section{References}

[1] Aase, K.K. (1999). An equilibrium model of catastrophe insurance futures and spreads. The Geneva Papers on Risk and Insurance Theory 24, 69-96.

[2] Barrieu, P., Bensusan, H., El Karoui, N., Hillairet, C., Loisel, S., Ravanelli, C. and Salhi, Y. (2012). Understanding, modelling and managing longevity risk: key issues and main challenges. Scandinavian Actuarial Journal 3, 203-231.

[3] Battocchio, P., Menoncin, F. and Scaillet, O. (2007). Optimal asset allocation for pension funds under mortality risk during the accumulation and decumulation phases. Ann. Oper. Res 152(1), 141-165. 
[4] Bauer, D., Börger, M. and Ruß, J. (2010). On the pricing of longevity-linked securities. Insurance: Mathematics and Economics 46, 139-149.

[5] Biagini, F., Rheinländer, T. and Widenmann, I. (2013). Hedging mortality claims with longevity bonds. ASTIN Bulletin 43(2), 123-157.

[6] Blake, D., Cairns, A., Dowd, K. and MacMinn, R. (2006). Longevity bonds: financial engineering, valuation, and hedging. The Journal of Risk and Insurance 73, 647-672.

[7] Cairns, A. (2010). Some notes on the dynamics and optimal control of stochastic pension fund models in continuous time. ASTIN Bulletin 30(1), 19-55.

[8] Christiansen, M.C. and Helwich, M. (2008). Some further ideas concerning the interaction between insurance and investment risks. Blätter DGVFM 29, 253266.

[9] Christensen, C.V. and Schmidli, H. (2000). Pricing catastrophe insurance products based on actually reported claims. Insurance Math. Econom. 27, 189-200.

[10] Cox, S.H., Fairchild, J.R. and Pedersen, H.W. (2000). Economic aspects of securitization of risk. ASTIN Bull. 30, 157-193.

[11] Cox, S.H. and Pedersen, H.W. (2000). Catastrophe risk bonds. North Amer. Actuarial J. 4(4), 56-82.

[12] Cummins, J.D. and Geman, H. (1993). An Asian option approach to the valuation of insurance futures contracts. Review Futures Markets 13, 517-557.

[13] Dahl, M, and Møller, T. (2006). Valuation and hedging of life insurance liabilities with systematic mortality risk. Insurance: Mathematics and Economics 39(2), 193-217.

[14] Dahl, M., Melchior, M. and Møller, T. (2008). On systematic mortality risk and risk-minimization with survivor swaps. Scandinavian Actuarial Journal, $114-146$.

[15] Delong, L., Gerrard, R. and Haberman, S. (2008). Mean-variance optimization problems for an accumulation phase in a defined benefit plan. Insurance Math. Econom. 42(1), 107-118.

[16] Embrechts, P. and Meister, S. (1995). Pricing insurance derivatives, the case of CAT-futures. Paper presented at the Symposium on Securitization of Insurance Risk, Atlanta, May 25-26.

[17] Menoncin, F. (2008). The role of longevity bonds in optimal portfolios. Insurance Math. Econom. 42(1), 343-358. 
[18] Menoncin, F. and Regis, L. (2017). Longevity-linked assets and pre-retirement consumption/portfolio decisions. Insurance: Mathematics and Economics, $\mathbf{7 6}$, $75-86$.

[19] Schilling, K., Bauer, D., Christiansen, M.C. and Kling, A. (2015). Decomposing life insurance liabilities into risk factors. Working Paper, University of Ulm.

[20] Schmidli, H. (2003). Modelling PCS options via individual indices. Working paper 187, Laboratory of Actuarial Mathematics, University of Copenhagen.

[21] Schmidli, H. (2008). Stochastic Control in Insurance. Springer-Verlag, London.

[22] Schmock, U. (1999). Estimating the value of the Wincat coupons of the Winterthur insurance convertible bond: a study of the model risk. ASTIN Bull. 29, $101-163$.

[23] Schradin, H.R. and Timpel, M. (1996). Einsatz von Optionen auf den PCS-Schadenindex in the Risikosteuerung von Versicherungsunternehmen. Mannheimer Manuskripte zu Versicherungsbetriebslehre, Mannheim, in German. 